\title{
LOW EXTRACELLULAR SODIUM PROMOTES ADIPOGENIC COMMITMENT OF HUMAN MESENCHYMAL STROMAL CELLS: A NOVEL MECHANISM FOR CHRONIC HYPONATREMIA-INDUCED BONE LOSS
}

Deledda C*, Fibbi B*, Benvenuti S*, Giuliani C*, Luciani P*, Monici M $^{\circ}$, Mazzanti B\#, Ballerini C§, Peri A*.

${ }^{*}$ Endocrine Unit, and ${ }^{\circ}$ ASAcampus Joint Laboratory, ASA Research Division, "Center for Research, Transfer and High Education on Chronic, Inflammatory, Degenerative and Neoplastic Disorders for the Development of Novel Therapies" (DENOThe), Dept. of Experimental and Clinical Biomedical Sciences "Mario Serio", University of Florence, Florence, Italy

"Haematology Unit, Department of Experimental and Clinical Medicine, University of Florence, Florence, Italy, ${ }^{\S}$ Department of NEUROFARBA, University of Florence, Florence, Italy

\section{BACKGROUND}

Hyponatremia represents an independent risk factor for osteoporosis and fractures, affecting both bone density and quality. A direct stimulation of osteoclastogenesis and bone resorption in the presence of reduced extracellular sodium concentrations $\left(\left[\mathrm{Na}^{+}\right]\right)$has been shown, but, to date, the effects of reduced $\left[\mathrm{Na}^{+}\right]$on osteoblasts have not been elucidated.

This study investigated the effects of a chronic reduction of extracellular $\left[\mathrm{Na}^{+}\right]$, independently of osmotic stress, on human mesenchymal stromal cells (hMSC) from bone marrow, which represent the common progenitor for osteoblasts and adipocytes.

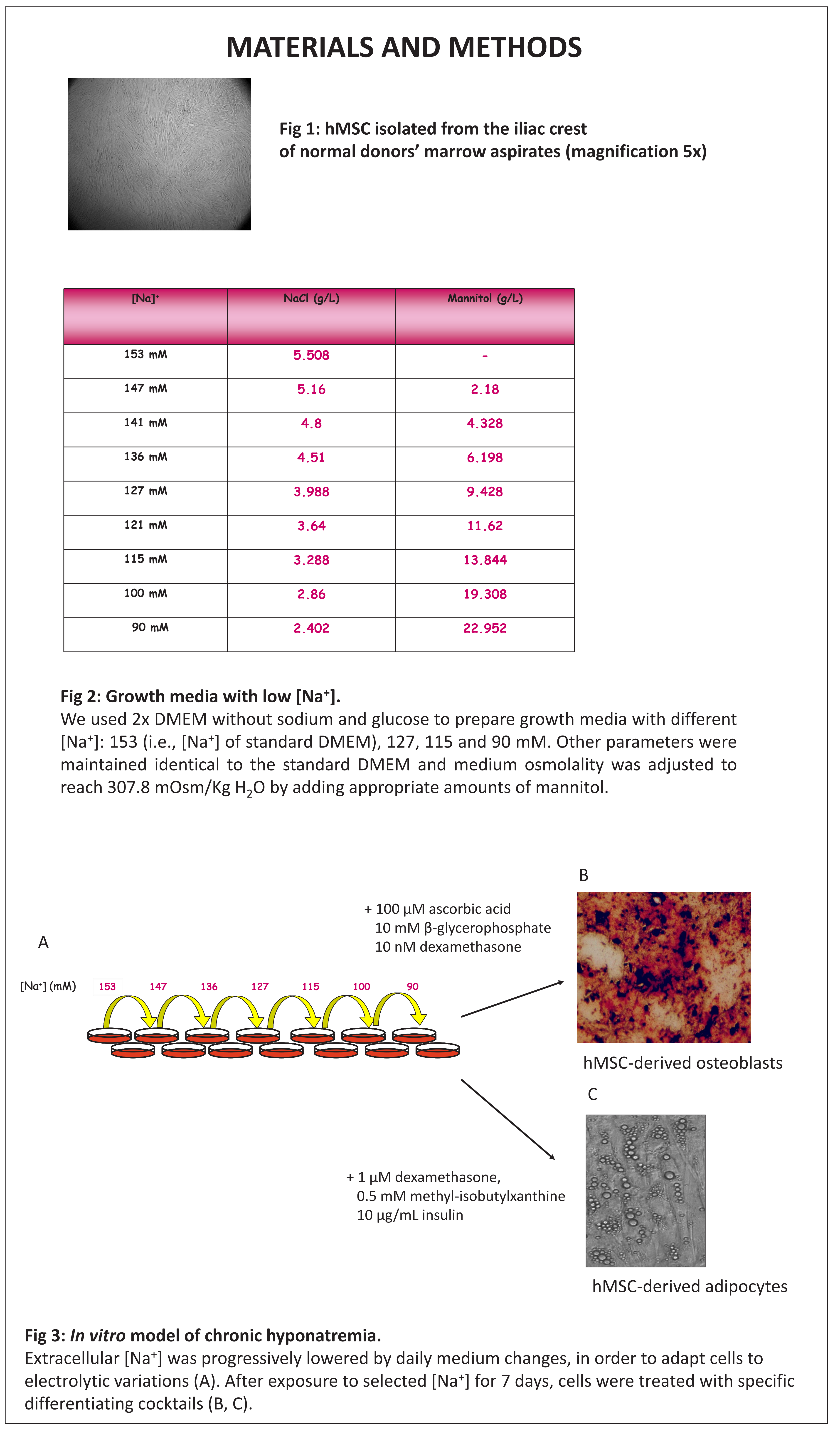

\section{RESULTS}

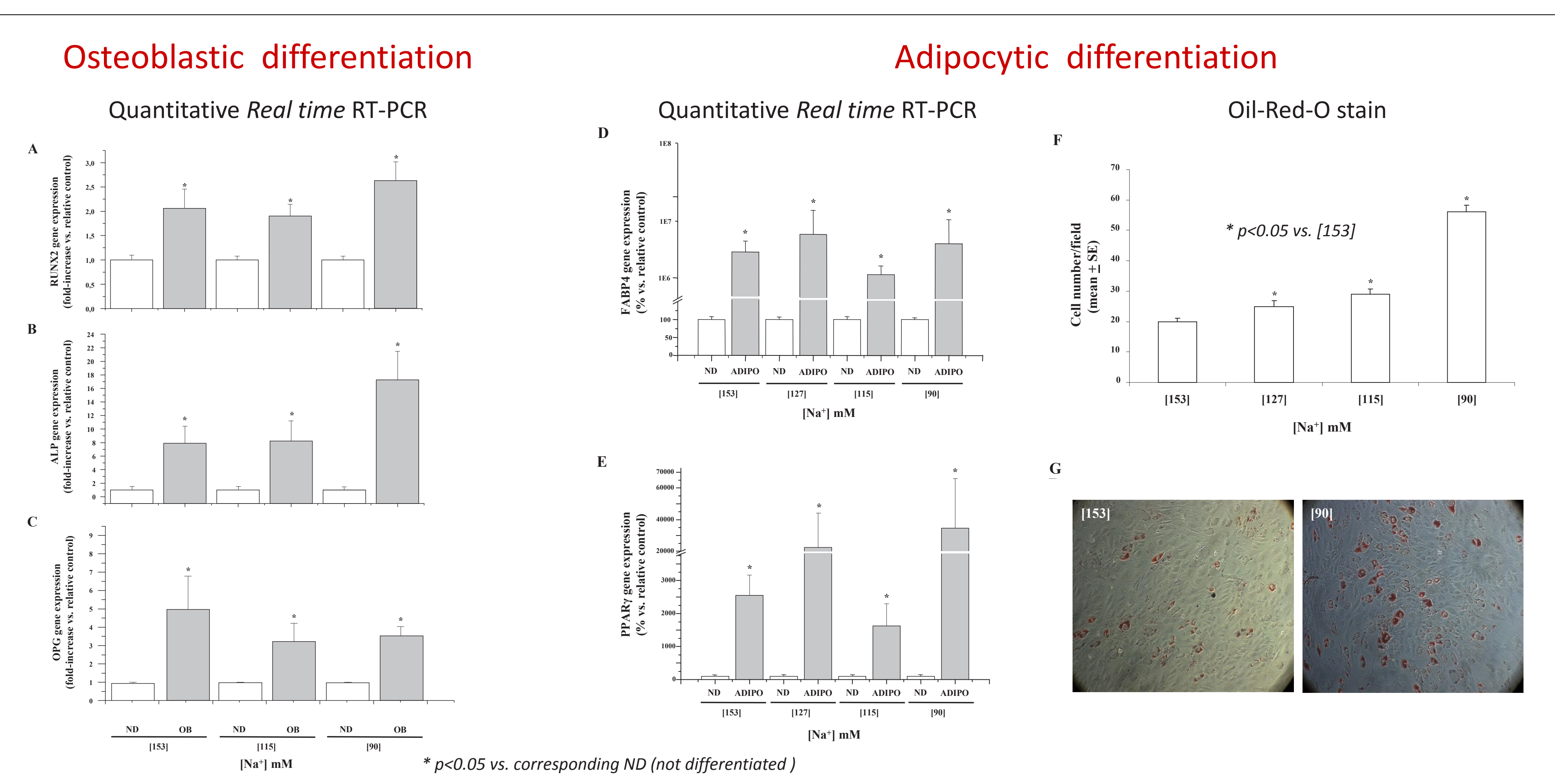

Fig 4: Effects of low extracellular [ $\left.\mathrm{Na}^{+}\right]$on hMSC commitment toward the osteogenic and adipogenic phenotype. At all [Na+] tested the gene expression of the osteogenic markers RUNX2 (A), ALP (B) and OPG (C) significantly increased after induction of hMSC to osteogenic differentiation. In differentiated cells, the expression of the three markers was not altered by exposure to low [ $\mathrm{Na}^{\mathrm{a}}$ ]. Similar results were obtained for the markers FABP4 (D) and PPARY (E) after adipogenic induction. This finding indicates that chronic hyponatremia does not
alter the differentiation potential of these cells. In addition, we performed a functional evaluation of the effect of reduced [Na+] on adipogenic differentiation of hMSC by Oil-Red-O stain (G) and adipose cell count by optical microscopy (F). The dose-dependent increase in the number of adipocytes as a function of reduced extracellular [ $\left.\mathrm{Na}^{+}\right]$suggests a preferential commitment toward the adipogenic phenotype. Magnification $5 \mathrm{x}_{\mathrm{f}}$
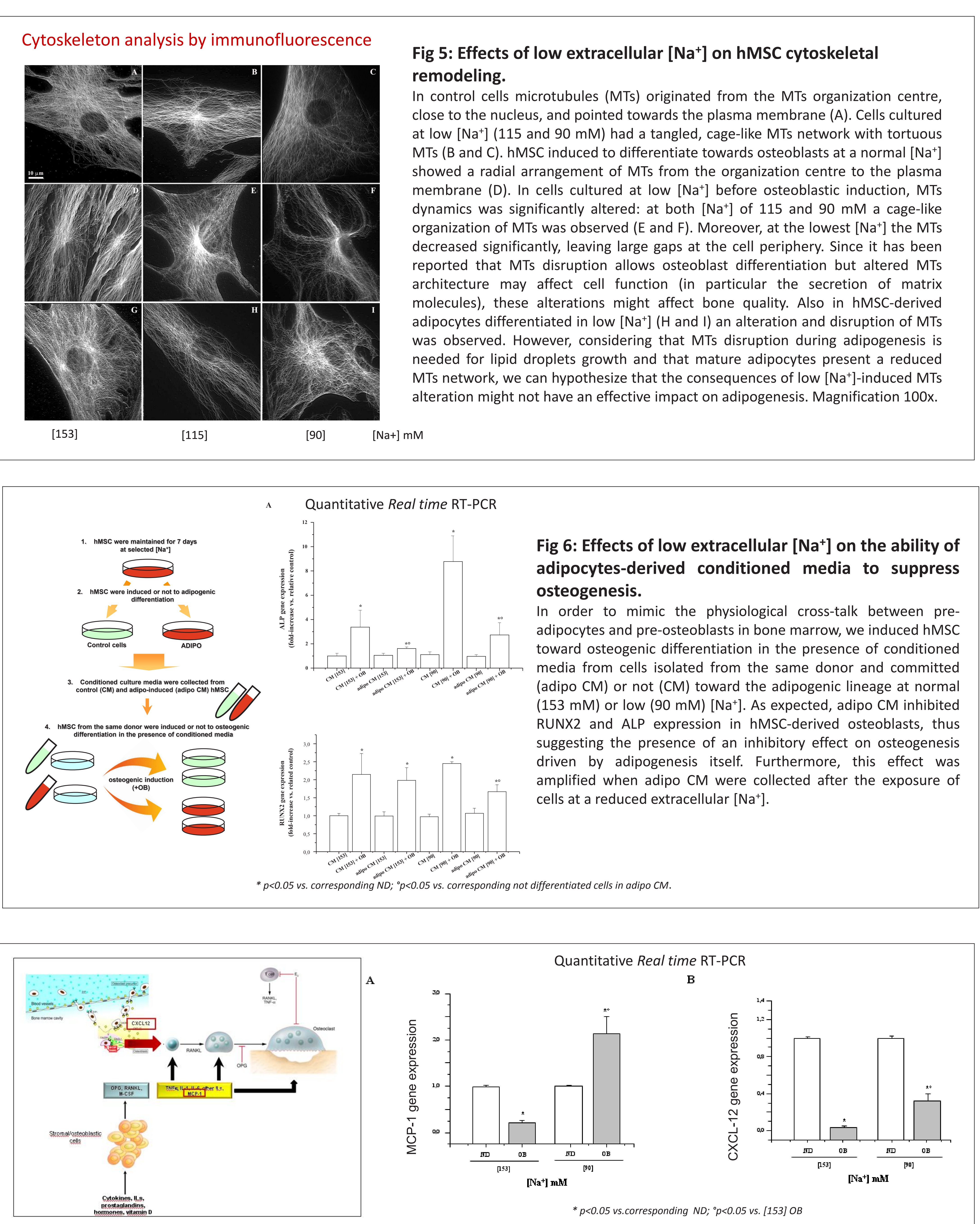

Fig 7: Effects of low extracellular [ $\left.\mathrm{Na}^{+}\right]$on osteoblast production of molecules able to modulate osteoclastogenesis and osteoclast activity. Low extracellular [Na+] reverted the inhibition of MCP-1 expression in hMSC-derived osteoblasts and attenuated the inhibition of the expression of the CXCL-12/SDF-1 gene. These data suggest that chronic hyponatrenta promses

\section{CONCLUSIONS}

We have demonstrated that in our in vitro model low $\left[\mathrm{Na}^{+}\right]$does not alter the intrinsic ability of hMSC to differentiate along the osteogenic and the adipogenic lineages, even if it associated with a cytoskeleton disorganization that could affect cell function. In vivo, mesenchymal precursors are exposed to conflicting stimuli, which determine a fine equilibrium between adipogenesis and osteogenesis. Our data suggest that reduced extracellular [ $\left.\mathrm{Na}^{+}\right]$alter this balance and promotes preferentially adipogenesis, by increasing both the number of precursors able to differentiate into adipocytes, and the production of soluble factors which inhibit osteoblastogenesis and induce osteoclastogenesis. These findings add new evidence suggesting that hyponatremia should be carefully taken into account by clinicians because of its negative effects on bone, in addition to the known neurological effects, and indicate for the first time that impaired osteogenesis appears to be involved, in addition to increased osteoclastogenesis and resorptive activity. 